\title{
A sliding gas-delivery system and ventilating chamber for small animals fixed in standard stereotaxic instruments
}

\author{
NELSON L. FREEDMAN \\ Queen's University, Kingston, Ontario, Canada
}

\begin{abstract}
An adjustable device for delivering volatile anesthetics or respiratory gases during stereotaxic surgery is described. The device provides for respiration on delivery and recovery of respired gases without interfering with cranial surgery or sacrificing stereotaxic accuracy.
\end{abstract}

Anesthesia and attendant respiratory problems introduce several complications in small-animal neurosurgery. Anesthesia must accompany placement of experimental animals in the stereotaxic instrument, yet anesthetics commonly depress respiratory centers in the brain and may lead to hypoventilation, acidosis, and hypoxia (e.g., Fink, Hanks, Ngai, \& Papper, 1963). Moreover, the design of most stereotaxic instruments precludes the use of anesthetic gases. Such problems might be avoided or circumvented if the animal could be artificially respirated until the anesthetic wears off or if a gas-delivery device could be attached to the stereotaxic instrument. Simple respiratory procedures are incompatible with the upper incisor bars and nose clamps normally provided, for example, with the Stoelting Lab Standard Stereotaxic Model 51600, Kopf Model 900 Small Animal Stereotaxic Instrument or Model 400 student version, and other more sophisticated models commonly found in experimental surgeries. For acute preparations when animals are sacrificed after surgery, a solution might be to perform a tracheotomy or insert an endotracheal tube expressly to respirate or deliver gases to the animal and preclude any of the above problems. However, there is some risk involved with the procedures. For example, recovery from tracheotomy can have serious consequences for the chronic preparations frequently used by behavioral neuroscientists, and endotracheal tubes can damage and possibly infect sensitive tissue in the respiratory passageways. I describe here a simple aluminum fitting for the upper incisor bar that replaces the snout clamp, preserves the location of the head in threedimensional space, and serves as a gas-mixing and ventilation chamber that avoids the above problems. Moreover, this fitting can be adapted to accomodate any commercially available small-animal respirator.

A modified version of this article appeared in the trade journal Kopf Carrier, No. 2, June 1989. Correspondence should be addressed to N. L. Freedman, Department of Psychology, Queen's University, Kingston, ON, Canada K7L 3N6.

\section{Apparatus}

Figure 1 shows the respirator fitting in front, bottom, and isometric views projection. The fitting is machined from a solid $30 \times 29 \times 26 \mathrm{~mm}$ block of aluminum. The fitting features a $16 \times 7 \times 29 \mathrm{~mm}$ channel (1) designed to slide smoothly over the tooth plate (upper incisor bar) with the nose clamp removed. The ventilation chamber (2), a truncated conical hole (15 mm outer diameter and $9 \mathrm{~mm}$ inner diameter), is drilled $23 \mathrm{~mm}$ into the front of the block parallel to the sliding channel to snugly accommodate the rat's snout and to provide room for air or volatile anesthetics to be delivered to the rear of the chamber. The frontal edge of the aperture is polished to prevent injury to the sensitive tissue of the snout. A notch (3) is cut between the front edge of the aperture and the sliding channel to fit around the rat's upper incisor, which fits into the tooth plate on most stereotaxic instruments to fix the animal in three-dimensional space. Two 4-mmdiameter brass tubes (4) extend into the side of the respiratory chamber, allowing pulses of air or volatile gases to be delivered to the posterior part of the chamber and ex-

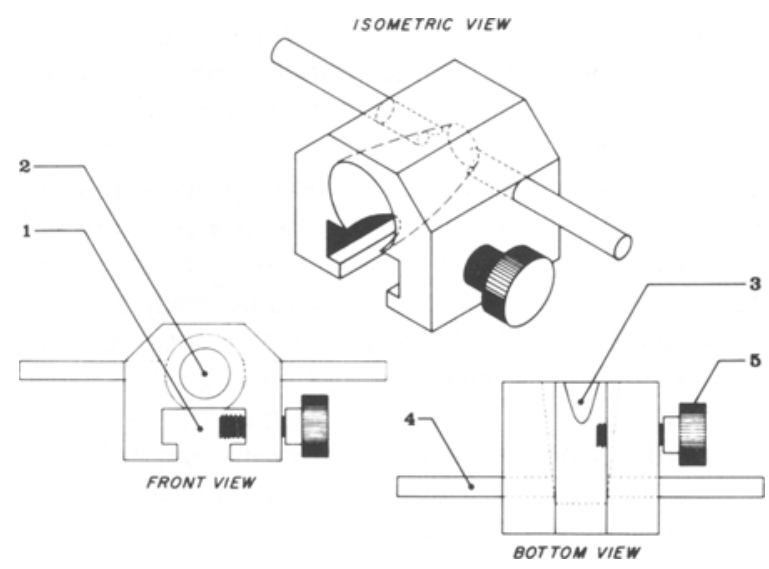

Figure 1. Schematic illustration of the ventilation adaptor in front, bottom, and isometric views: (1) Channel fitting over tooth plate. (2) Ventilation chamber. (3) Upper incisor notch. (4) Brass tubes to respirator. (5) Manual set screw. 
pired gases to be removed. Finally, a knurled knob (5) is used as a manual set screw to rigidly hold the adaptor in place on the tooth plate over the snout and upper incisor.

\section{Method of Use}

The rat is first anesthetized with an appropriate dose of anesthetic (I use Nembutal or Somnotal, $65 \mathrm{mg} / \mathrm{kg}$ i.p.). To prevent aspiration of excess saliva into the lungs, atropine sulfate or atropine methyl nitrate may be given as required at this time. I find, however, that the forced air passing through the respiratory passages normally provides sufficient drying. Typically, the rat's scalp is shaved and the ear bars are set in the external auditory meatus and centered in the stereotaxic instrument. At this point, the entire upper incisor bar is retracted and the ventilation chamber is set to the rear of the tooth plate (Figure 2A). As usual, the entire upper incisor unit is moved along its anterior-posterior travel to fit the upper incisors into the rectangular hole in the tooth plate (Figure 2B). The unit is normally retracted slightly and then screwed tightly to the frame to rigidly fix the head in threedimensional space. The tongue is then gently retracted with a blunt forceps to ensure that the oral passages are not blocked.

As a last step, the ventilator unit is slid across the tooth plate to fit snugly on the snout. The thumb screw is then tightened to rigidly fix the unit to the plate (Figure $2 \mathrm{C}$ ). A thin film of petroleum jelly on the tooth plate helps the chamber slide smoothly and provides a tight seal. If the ventilation chamber is not snug, air and/or gases escape and do not register on pressure monitors.

Any of several small-animal respirators such as the Stoelting Small Rodent Respirator or the Ugo Basile Rodent Ventilator may be used with the ventilating chamber. The respirator is normally attached to the inspiration-expiration tubes using appropriate-sized Tygon tubing. If a continuous slow flow of anesthetic gases is required, commercially available gas anesthesia systems (Stoelting Lab) for halothane or isoflurane administration can be easily adapted to the ventilator unit. We control and monitor respiration rate and pressure with an $E$ \& $M$ Instruments Small Animal Respirator. The inspirationexpiration ratio is set to 1 , and the respiration rate is set at 30 or more pulses per minute with a pressure equal to $10-12 \mathrm{~cm}$ of water. If the system is open, the pressure drops to zero, and the fitting should be readjusted more snugly on the snout. Respiration rate is also confirmed by watching the rib cage rise and fall with the pulses of the machine. If the animal is breathing on its own, the rates will not match; snug adjustment around the snout or sealing the chamber around the snout with petroleum jelly may correct this. ${ }^{1}$ Occasionally, excess air is swallowed and produces noticable stomach distension. This can be avoided by reducing the pulse pressure.

On completion of surgery, the procedure is reversed; the thumbscrew is loosened, the chamber is slid back, and the respirator or gas anesthesia device is turned off. Respiration should be observed for several minutes before removal from the frame to make sure the animal is breath-
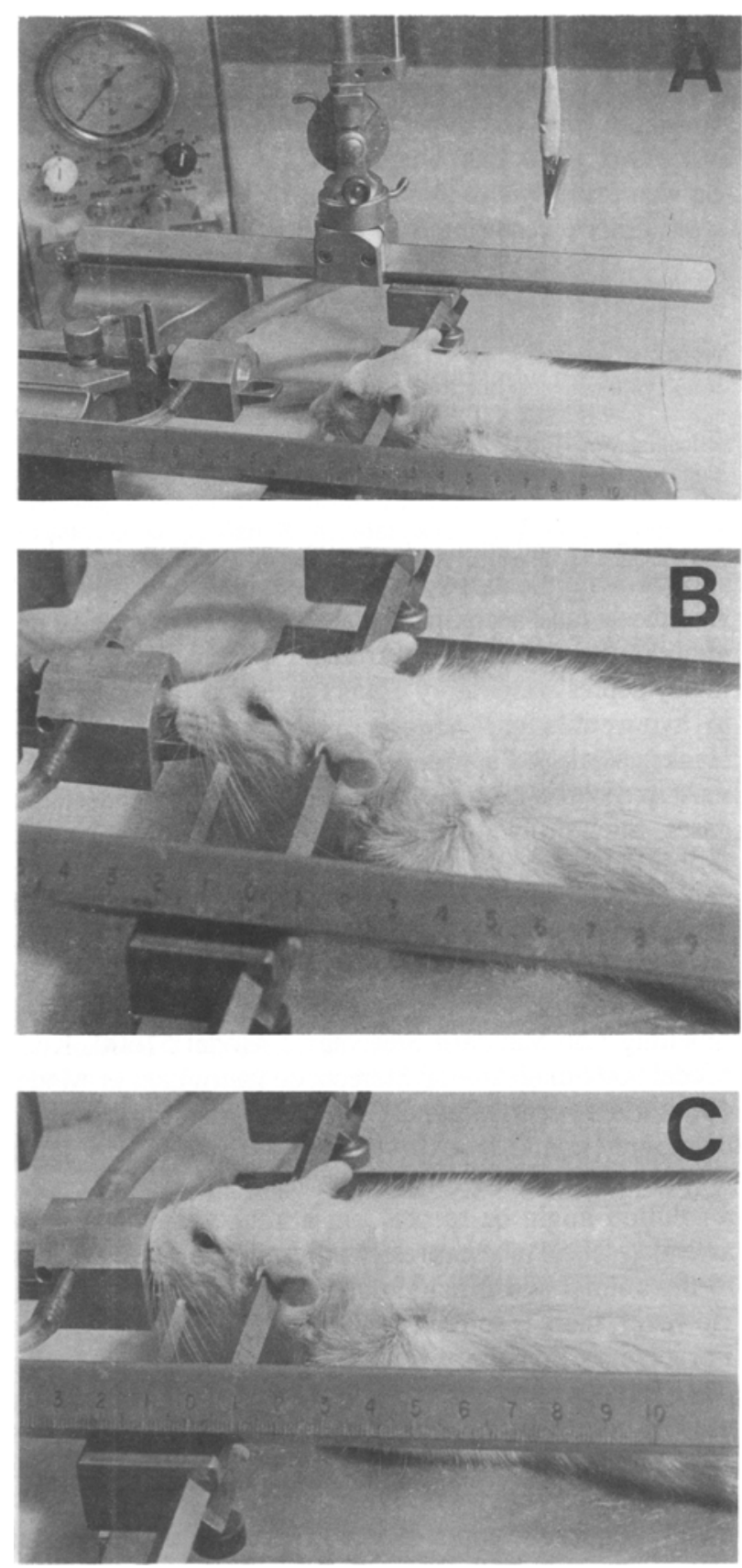

Figure 2. The rat is fixed in the ear hars with the tooth plate and ventilation chamber in the retracted position $(A)$, with the upper incisors in position (B), and with the ventilation chamber fixed over the snout (C).

ing on its own. If respiration is shallow, the chamber and respirator can be easily reattached. If necessary, we observe the animal closely until it recovers muscle tone, at which time it is removed from the frame.

\section{Discussion}

The sliding ventilation chamber provides a simple and effective means for respirating or delivering volatile sub- 
stances to small animals without the additional surgical intervention and trauma of tracheotomy, and it also substitutes for the nose clamp normally used to rigidly fix the snout to the tooth clamp. The procedure is no more difficult to use than the standard nose clamp, but provides for fast delivery of gas anesthetics or artificial respiration until the animal recovers from the anesthetic. The device also affords an extra dimension of safety and humane concern for recovery from anesthetic during smallanimal stereotaxic neurosurgery.

\section{REFERENCE}

Fink, B. R., Hanks, E. C., Ngai, S. H., \& Papper, E. M. (1963). Central regulation of respiration during anesthesia and wakefulness. Annals of the New York Academy of Sciences, 109, 898.

\section{NOTE}

1. J. Steinmetz (personal communication, February 1992) suggested that a doughnut-shaped rubber curtain, similar to that used by dentists, glued to the front of the chamber may provide the snug fit. 